\title{
Class Groups and General Linear Group Cohomology for a Ring of Algebraic Integers
}

\author{
Stephen A. Mitchell*
}

December 1994

Note: This is a revised and expanded version of an earlier (and much shorter) preprint with the same title. The main theorems and their proofs are unchanged, but much exposition has been added.

Suppose that $\mathrm{F}$ is a number field, with ring of integers $\mathcal{O}_{F}$. Let $\ell$ denote an odd prime and let $R=\mathcal{O}_{F}[1 / \ell]$. In [3], the author and W.Dwyer gave an explicit conjectural computation of the mod $\ell$ cohomology of the infinite general linear group GLR. Here is the quickest and simplest statement of the conjecture (all homology and cohomology groups have $\mathbb{Z} / \ell$ coefficients): let $\mathrm{U}$ denote the infinite unitary group. Let $J_{a}^{*}$ denote the mod $\ell$ cohomology of the homotopy-fibre of the $\ell^{a}$-th power map $S U \rightarrow S U$. Thus as an algebra, $J_{a}^{*}$ is the tensor product of a certain polynomial algebra $P_{a}^{*}$ and a companion exterior algebra $E_{a}^{*}$. An explicit description is given in Section 1; here we just remark that the Hopf algebra $P_{a}$ dual to $P_{a}^{*}$ is $H_{*} B U /\left(\ell^{a}\right.$-th powers $)$. Let $\mathcal{P}$ denote the algebra of Steenrod $\ell$-th power operations.

Conjecture A. Suppose F contains the $\ell$-th roots of unity $\mu_{\ell}$. Then as $\mathcal{P}$-Hopf algebras

$$
H^{*}(G L R ; \mathbb{Z} / \ell) \cong\left(H^{*} B U\right)^{s} \otimes\left(H^{*} U\right)^{r_{2}+s} \otimes J_{a_{1}}^{*} \otimes \ldots \otimes J_{a_{m}}^{*}
$$

where $r_{2}=\frac{1}{2}[F: \mathbb{Q}]$, $\mathrm{s}$ is the number of primes dividing $\ell$ in $\mathcal{O}_{F}$, and the $\ell$-torsion subgroup of Pic $\mathrm{R}$ is $\mathbb{Z} / \ell^{a_{1}} \oplus \ldots \oplus \mathbb{Z} / \ell^{a_{m}}$.

\footnotetext{
*Supported by a grant from the National Science Foundation
} 
The assumption on $\ell$-th roots of unity is made only to simplify the statement. In the general case the conjecture is formulated by setting $F_{0}=F\left(\mu_{\ell}\right)$, $R_{0}=\mathcal{O}_{F_{0}}(1 / \ell)$. Then $H^{*} G L R=\left(H^{*} G L R_{0}\right) \Delta_{F}$, where the fixed points of $\Delta_{F}=G\left(F\left(\mu_{\ell}\right) / F\right)$ are taken in an abelian category of bicommutative Hopf algebras. A recipe for carrying out this fixed-point construction can be found in Section 1.

There is another way of stating the conjecture that is slightly more complicated, but more enlightening. In order to avoid technical distractions, explicit description of the $\Delta_{F}$-action is postponed to Section 1 . Let $\mu\left(R_{0}\right)$ denote the $\ell$-torsion subgroup of $R_{0}^{\times}$.

Conjecture $A^{\prime} . H^{*} G L R \cong A \otimes B \otimes C \otimes D$ as $\mathcal{P}$-Hopf algebras, where

1. The structure of $\mathrm{A}$ depends only on $\mu\left(R_{0}\right)$ as $\Delta_{F}$-module (or on the orders of $\Delta_{F}$ and $\left.\mu\left(R_{0}\right)\right)$. In fact $A \cong H^{*} G L \mathbb{F}$ for a suitable choice of residue field $\mathbb{F}$ of $\mathrm{R}$, where $H^{*} G L \mathbb{F} \cong\left(H^{*} B U \otimes H^{*} U\right)^{\Delta_{F}}$.

2. The structure of $\mathrm{B}$ depends only on the units $R_{0}^{\times} \bmod$ torsion as $\Delta_{F^{-}}$ module. In fact $B \cong\left(H^{*} U\right)^{r_{2}} \otimes\left(H^{*} U / O\right)^{r_{1}}$, where $r_{2}$ is one-half the number of non-real embeddings of $\mathrm{F}, r_{1}$ is the number of real embeddings, and $\mathrm{O}$ is the infinite orthogonal group. In particular $\mathrm{B}$ is an exterior algebra.

3. The structure of $\mathrm{C}$ depends only on the $\ell$-torsion subgroup of Pic $R_{0}$ as $\Delta_{F}$-module. In fact $C \cong\left(J_{a_{1}}^{*}\right)_{F} \otimes \ldots \otimes\left(J_{a_{m}}^{*}\right)_{F}$, where the $a_{i}$ are as above and the $\Delta_{F}$-action on the individual factors arises from choosing a basis of $\Delta_{F}$-eigenvectors for $\mathrm{Pic} R_{0} / \ell$.

4. The structure of $\mathrm{D}$ depends only the set $S_{0}$ of primes dividing $\ell$ in $\mathcal{O}_{F_{0}}$ as $\Delta_{F}$-set. In fact $\mathrm{D}$ is a tensor product of $\left(s_{0}-1\right) \mathcal{P}$-Hopf algebras of the form $\left(H^{*} B U \otimes H^{*} U\right)^{\Delta_{F}}$, where $s_{0}=\left|S_{0}\right|$ and the $\Delta_{F}$-action on the individual factors arises by choosing a basis of $\Delta_{F}$-eigenvectors for the reduced permutation module $\mathbb{Z} \bar{S}_{0}=\operatorname{Ker}\left(\mathbb{Z} / \ell\left[S_{0}\right] \rightarrow \mathbb{Z} / \ell\right)$.

Let $T B r R$ denote the Tate module of the Brauer group of $\mathrm{R}$ :

$$
\operatorname{TBr} R=\operatorname{Hom}\left(\mathbb{Z} / \ell^{\infty}, \operatorname{Br} R\right)
$$


Then $T B r R_{0} \cong Z_{\ell} \bar{S}_{0}$ as $\Delta_{F}$-module, so $\mathrm{D}$ can also be regarded as corresponding to the Brauer group.

The factor A in Conjecture $A^{\prime}$ is in fact a retract of $H^{*} G L R$, because $B G L \mathbb{F}^{+}$is a homotopy retract of $B G L R^{+}$, after localization at $\ell[5]$. However the reader is cautioned that the decomposition of Conjecture $A^{\prime}$ will not, in general, reflect a product decomposition of $B G L R^{+}$.

The equivalent conjectures $\mathrm{A}$ and $A^{\prime}$ would follow immediately from one formulation of the Lichtenbaum-Quillen conjectures. Namely, the LichtenbaumQuillen conjectures assert that there is a homotopy equivalence

$$
\left(B G L R^{+}\right)^{\wedge} \cong Y_{R}
$$

for a certain space $Y_{R}$. This space is equivalent to the étale $\mathrm{K}$-theory space of Dwyer and Friedlander [1], but we will define it as the basepoint component of the zero-th space of a certain Bousfield localization of the spectrum KR (Section 3). These two descriptions are equivalent by a theorem of Thomason [14]. The cohomology of $Y_{R}$ is computed in [3], where it is shown to have exactly the form stated in the above conjectures.

The Lichtenbaum-Quillen conjectures themselves still appear to be inaccessible. However it would be very interesting to even prove that the induced map $H^{*} Y_{R} \rightarrow H^{*} B G L R$ is injective. The present paper is a first step in that direction, focusing on the polynomial part of $H^{*} Y_{R}$. Write $P^{*}\left(\Delta_{F}, \hat{K}_{0} R_{0}\right)$ for the polynomial part of the algebras $\mathrm{A}$ and $\mathrm{C}$ in Conjecture $A^{\prime}$ (this notation is explained in Section 1). Thus $P^{*}\left(\Delta_{F}, \hat{K}_{0} R_{0}\right) \cong\left(H^{*} B U \otimes P_{a_{1}}^{*} \otimes \ldots \otimes P_{a_{m}}^{*}\right)^{\Delta_{F}}$ for a certain $\Delta_{F}$ action.

We prove:

Theorem 1.6. There is a retraction as $\mathcal{P}$-Hopf algebras

$$
\phi^{*}: H^{*} G L R \longrightarrow P^{*}\left(\Delta_{F}, \hat{K}_{0} R_{0}\right)
$$

Combining this with results of Quillen [12] leads to an interesting corollary. Call an element of $H^{*} G L$ nil if its restriction to every $G L_{n}$ is nilpotent $(n<\infty)$.

Theorem 2.1. $\phi^{*}$ induces an isomorphism $H^{*} G L R /($ nil elements $)=P^{*}\left(\Delta_{F}, \hat{K}_{0} R_{0}\right)$. 
Of course there may exist elements which are nil but not nilpotent. Indeed the results of [3] predict very precisely the extent to which this occurs. For example, assuming the Lichtenbaum-Quillen conjectures, every nil element is nilpotent if and only if there is a unique prime dividing $\ell$ in $\mathcal{O}_{F_{0}}$. This phenomenon is discussed further in Section 5.

Here is a brief sketch of the proofs of these theorems. For simplicity we assume $\mu_{\ell} \subset R$. Most of the time we work in homology rather than cohomology. Consider the Hopf algebra summand $\mathrm{P}\left(\hat{K}_{0} R\right)$ (dual to $\left.P^{*}\left(\hat{K}_{0} R\right)\right)$ in $H_{*} Y_{R}$. We show that it is generated by certain maps $f_{\alpha}: B \mu(R) \rightarrow Y_{R}$, where $\alpha$ ranges over a generating set of $\hat{K}_{0} R$. Here "generated by" means generated as an algebra by the images of the even-dimensional generators of $H_{*} B \mu$. On the other hand it will follow immediately from the construction that each $f_{\alpha}$ lifts to $B G L R^{+}$, and this leads to the desired homology retraction.

In fact our map $P\left(\hat{K}_{0} R\right) \rightarrow H_{*} B G L R$ is easy to construct directly, and we do so in Section 1. The dual map $\left.H^{*} G L R \rightarrow P^{*}\left(\hat{K}_{0} R\right)\right)$ is the map that enters into Theorem 1.6. This map can be interpreted as a sort of restriction (modulo nilpotent elements) to all possible maximal $\ell$-tori in $G L_{n} R, n \geq 1$. Now Quillen showed that if a class in $H^{*} G L_{n} R$ restricts to zero on every maximal $\ell$-torus, then it is nilpotent. From this one easily concludes that the kernel of $\phi^{*}$ is precisely the ideal of nil elements. An interesting point here is that although $G L_{n} R$ typically has many conjugacy classes of maximal $\ell$ tori, in effect a single such $\ell$-torus suffices to detect non-nil stable cohomology classes. However it is not at all apparent, a priori, that $\phi^{*}$ is surjective. This is where Theorem 1.6 comes in.

The paper is organized as follows. In section 1 we introduce some general facts about Hopf algebras, describe the algebras $P_{a}$ explicitly, and state the main result Theorem 1.6. Section 2 shows how to combine Theorem 1.6 with Quillen's work to get Theorem 2.1. We also show how Quillen's unstable classes in $H^{*} G L_{n} R$ ([12] , section 14) are constructed from this point of view. The rather long Section 3 is partly expository. Its aim is to make the technical intricacies of [3] more accessible; some of these technical details are needed for the proof of Theorem 1.6. The proof of Theorem 1.6 then appears in Section 4. In section 5 we indulge in some speculative remarks concerning the remaining non-nilpotent Hopf summand in Conjecture $A^{\prime}$, and its relation to the Brauer group. 
I would like to thank the referee for criticizing an earlier and much shorter manuscript, and especially for insisting that the exposition of [3] be expanded.

Notation. An odd prime $\ell$ is fixed throughout, and all homology and cohomology groups have $\mathbb{Z} / \ell$-coefficients. $\mathcal{A}$ denotes the mod $\ell$ Steenrod algebra, and $\mathcal{P}$ is the subalgebra generated by the $\ell$-th power operations; thus $\mathcal{P} \cong \mathcal{A} / \mathcal{A} \beta \mathcal{A}$, where $\beta$ is the Bockstein. Spectra and spaces are implicitly localized at $\ell$. Notations such as $\hat{P} i c R,\left(B G L R^{+}\right)^{\wedge}$, $\hat{\mathcal{K}}$ always indicate $\ell$ adic completion of the group, space or spectrum, as the case may be. The following notation is also fixed throughout the paper:

Roots of unity. $\mu_{\ell^{n}}$ denotes the group of $\ell^{n}$-th roots of unity in $\mathbb{C}, 1 \leq n \leq \infty$. We also write $\mu(A)$ for the group of $\ell$-power roots of unity in a given integral domain $\mathrm{A}$; if $A=R_{0}$ we abbreviate this further by setting $\mu=\mu\left(R_{0}\right)$. Let $\Gamma^{\prime}=A u t \mu_{\ell^{\infty}}, \Delta=A u t \mu_{\ell}, \Gamma=\operatorname{Ker}\left(\Gamma^{\prime} \rightarrow \Delta\right)$. Thus $\Gamma^{\prime}$ is canonically isomorphic to $Z_{\ell}{ }^{\times}$, and there is a uniquely split extension

$$
\Gamma \rightarrow \Gamma^{\prime} \rightarrow \Delta
$$

Composing the unique splitting map in this sequence with the canonical isomorphism $\Gamma^{\prime} \cong Z_{\ell}{ }^{\times}$yields the "Teichmuller character" $\omega: \Delta \rightarrow Z_{\ell}{ }^{\times}$. The pro-group ring $Z_{\ell}[[\Gamma]]$ is denoted by $\Lambda$. A choice of topological generator $\gamma$ of $\Gamma$ defines an isomorphism $\Lambda \cong Z_{\ell}[[T]]$, where $Z_{\ell}[[T]]$ is a power series ring and $\gamma \mapsto 1+T$. Similarly $\Lambda^{\prime}=Z_{\ell}\left[\left[\Gamma^{\prime}\right]\right]$; note $\Lambda^{\prime}=\Lambda[\Delta]$.

Topological K-theory. $\mathcal{K}$ is the periodic complex K-theory spectrum. The natural map $\{k \in \mathbb{Z}:(k, \ell)=1\} \rightarrow[\hat{\mathcal{K}}, \hat{\mathcal{K}}]$ sending $\mathrm{k}$ to the Adams operation $\Psi^{k}$ extends to a (continuous) injection $\Gamma^{\prime} \rightarrow[\hat{\mathcal{K}}, \hat{\mathcal{K}}]$. The latter map in turn induces an isomorphism $\Lambda^{\prime} \cong[\hat{\mathcal{K}}, \hat{\mathcal{K}}]$.

The cyclotomic tower. We assume $\mathrm{F}$ is a subfield of $\mathbb{C}$. Set $F_{0}=F\left(\mu_{\ell}\right)$, $F_{\infty}=F\left(\mu_{\ell^{\infty}}\right), R_{0}=\mathcal{O}_{F_{0}}[1 / \ell]$, etc. Let $\Delta_{F}=G\left(F_{0} / F\right)$ and let $d_{F}$ denote the order of $\Delta_{F}$. Note $\Delta_{F} \subset \Delta$. Let $a_{F}=\max \left\{a: \mu_{\ell^{a}} \subset F_{0}^{\times}\right\}$. Let $\Gamma_{F}$ (resp. $\left.\Gamma_{F}^{\prime}\right)$ denote $G\left(F_{\infty} / F_{0}\right)$ (resp. $G\left(F_{\infty} / F\right)$, so that $\Gamma_{F} \subset \Gamma, \Gamma_{F}^{\prime} \subset \Gamma^{\prime}$, $\Gamma_{F}^{\prime}=\Gamma_{F} \Delta_{F}$, etc. Write $\Lambda_{F}$ for the pro-group ring $Z_{\ell}\left[\left[\Gamma_{F}\right]\right]$, so $\Lambda_{F} \subset \Lambda$. Similarly $\Lambda_{F}^{\prime} \subset \Lambda^{\prime}$. Note that if we choose an isomorphism $\Lambda \cong Z_{\ell}[[T]]$ as above, $\Lambda_{F}$ corresponds to the subring $Z_{\ell}\left[\left[T_{F}\right]\right]$ with $T_{F}=(1+T)^{\ell^{a}}-1$. S (resp. $S_{0}$ ) is the set of primes over $\ell$ in $\mathcal{O}_{F}$ (resp. $\mathcal{O}_{F_{0}}$ ), and s (resp. $s_{0}$ is the 
cardinality of $\mathrm{S}\left(\right.$ resp. $\left.S_{0}\right) \cdot r_{2}$ (resp. $r_{2,0}$ ) denotes $1 / 2$ the number of nonreal embeddings of $\mathrm{F}$ (resp. $F_{0}$ ), and $r_{1}$ is the number of real embeddings of $\mathrm{F}$.

\section{Hopf Algebras, Class Groups and $H_{*} B G L R^{+}$}

In this section we define $P\left(\Delta_{F}, \hat{K}_{0} R_{0}\right)$ and define a map

$$
\phi: P\left(\Delta_{F}, \hat{K}_{0} R_{0}\right) \longrightarrow H_{*} B G L R^{+}
$$

whose dual is the map discussed in the introduction. At the end of the section we state our main result, Theorem 1.6, but postpone the proof to Section 4 . Related discussion of some of this material can be found in [10].

Let $\mathcal{H}$ denote the category of connected graded bicommutative Hopf algebras of finite type over $\mathbb{Z} / \ell$. This category is an abelian category [13], with coproducts given by tensor products of Hopf algebras. In fact the groups $\operatorname{Hom}_{\mathcal{H}}(A, B)$ are profinite abelian $\ell$-groups and in particular are modules over $Z_{\ell}$. We will need several refinements of $\mathcal{H}$ :

(i) If $\mathrm{G}$ is a profinite group, $\mathcal{H} \mathrm{G}$ is the category of G-modules in $\mathcal{H}$. Thus an object of $\mathcal{H} \mathrm{G}$ is an object $\mathrm{A}$ of $\mathcal{H}$ together with a continuous homomorphism

$G \rightarrow$ Aut $_{\mathcal{H}} A$. A morphism is just a morphism in $\mathcal{H}$ that commutes with the G-action.

(ii) $\mathcal{H} \mathcal{P}$ is the category of unstable $\mathcal{P}$-Hopf algebras $\mathrm{A}, A \in \mathcal{H}$. There is also a dual category $\mathcal{H} \mathcal{P}^{*}$ in which Steenrod operations go down instead of up. $\mathcal{H} \mathcal{A}$ and $\mathcal{H} \mathcal{A}^{*}$ are defined similarly.

(iii) $\mathcal{H} \mathcal{P} G$ is the category of unstable $\mathcal{P}[\mathrm{G}]$-Hopf algebras $\mathrm{A}, A \in \mathcal{H}$. Thus an object of $\mathcal{H P G}$ has both a $\mathrm{G}$-action as in (i) and a $\mathcal{P}$-action as in (ii), and these actions commute. $\mathcal{H} \mathcal{P}^{*} G, \mathcal{H} \mathcal{A G}$ and $\mathcal{H} \mathcal{A}^{*} G$ are defined similarly.

All of these categories are again abelian, with kernels and cokernels being computed in $\mathcal{H}$. Now recall that $\mathcal{H}$ splits as a product of smaller subcategories:

Remark 1.1 Every $A \in \mathcal{H}$ splits uniquely as $A=A^{+} \otimes A^{-}$where $A^{+}$ is concentrated in even degrees and $A^{-}$is an exterior algebra on primitive generators of odd degree. In fact this yields a splitting of categories $\mathcal{H} \cong$ $\mathcal{H}^{+} \times \mathcal{H}^{-}$. 
Remark 1.2 Every $A \in \mathcal{H}^{+}$splits uniquely as

$$
A=\otimes_{i=0}^{\ell-2} A[i]
$$

where $A[i]$ has both primitives and indecomposables concentrated in degrees $2 n$ with $n=i \bmod \ell-1$. Again this yields a splitting of categories $\mathcal{H}^{+} \cong \prod \mathcal{H}[i]$.

It is not hard to show that if $\mathrm{A}$ is an object in $\mathcal{H} \mathcal{P} \mathrm{G}$, both of these splittings are automatically splittings in $\mathcal{H P G}$.

We next discuss some fundamental examples. The most basic example of all is $S=H_{*} B U$, regarded as an object in $\mathcal{H P}^{*} \Gamma^{\prime}$ in the usual way: the $\mathcal{H} \mathcal{P}^{*}$ structure arises from the $\mathrm{H}$-space structure on $\mathrm{BU}$, and the $\Gamma^{\prime}$-action arises from the canonical identification of $\Gamma^{\prime}$ with the $\ell$-adic Adams operations. In purely algebraic terms, $\mathrm{S}$ is the symmetric algebra on the $\mathcal{P}$-coalgebra $\mathrm{B}$ with basis $\left\{b_{n}:\left|b_{n}\right|=2 n, n \geq 1\right\}$, coproduct $\Delta b_{n}=\sum_{i+j=n} b_{i} \otimes b_{j}$, and Steenrod operations given by a classical formula which we need not recall here. The $\Gamma$-action is trivial, but $\Delta$ acts on $\mathrm{S}[\mathrm{i}]$ as $\omega^{i}$. (For one way to see this last fact, compare [10], 3.3-3.7 and the remarks following.)

Now let $P_{a}$ denote the cokernel (in $\mathcal{H}$ ) of $\ell^{a} \cdot 1_{S}$. It is easy to see that $P_{a}$ is just $\mathrm{S}$ modulo the ideal generated by $\ell^{a}$-th powers. The dual Hopf algebra $P_{a}^{*}$ is a polynomial algebra on generators $y_{n, k}$ of dimension $2 n \ell^{k}$, where $\mathrm{n}$ is prime to $\ell$ and $0 \leq k<a$. Note $P_{a}$ is an object in $\mathcal{H P}^{*} \Delta$.

If $\mathrm{N}$ is a finitely-generated $Z_{\ell}$-module with rank s and exponents $\ell^{a_{1}}, \ldots, \ell^{a_{r}}$, we define an object $\mathrm{Q}(\mathrm{N})$ in $\mathcal{H} \mathcal{P}^{*} \Delta$ by

$$
Q(N)=\otimes^{s} S \otimes P_{a_{1}} \otimes \ldots \otimes P_{a_{r}}
$$

Now suppose $\Delta^{\prime}$ is a subgroup of $\Delta$, of order $\mathrm{d}$, and $\mathrm{N}$ is a $Z_{\ell} \Delta^{\prime}$-module. We will associate to $\mathrm{N}$ an object $\mathrm{P}(\mathrm{N})$ in $\mathcal{H} \mathcal{P}^{*} \Delta^{\prime}$. Although the construction is quite elementary, the $\Delta^{\prime}$-action gets a bit confusing so we illustrate it in Example 1.3 and Example 1.7. A key point to bear in mind here is that the $\Delta^{\prime}$-action we define is actually a blend of two distinct actions: an "external" action via Adams operations, which is inherited from the $\Delta$-action on $\mathrm{Q}(\mathrm{N})$ and has nothing to do with the $\Delta^{\prime}$-action on $\mathrm{N}$, and an "internal" or "Galois" action induced directly from the action on $\mathrm{N}$. We also need the notion of "Tate twisting". If $\mathrm{A}$ is any object in $\mathcal{H} \Delta^{\prime}$ the $\mathrm{i}$-th Tate twist $\mathrm{A}(\mathrm{i})$ has the same underlying Hopf algebra but with the twisted action $\sigma \cdot x=\omega^{i}(\sigma) \sigma(x)$ for 
$\sigma \in \Delta^{\prime}, x \in A$. Here $\omega: \Delta^{\prime} \rightarrow Z_{\ell} \times$ is the Teichmuller character (for further discussion, see the remarks on Tate twisting at the beginning of Section 3).

Let $N=\oplus_{i=0}^{d-1} N_{i}$ denote the $\Delta^{\prime}$-eigenspace decomposition. Then

$$
P(N)=\otimes_{i=0}^{d-1} Q\left(N_{i}\right)(i)
$$

We write $\mathrm{P}\left(\Delta^{\prime}, \mathrm{N}\right)$ for the $\Delta^{\prime}$-fixed points (in $\mathcal{H}$ ) of this action. To compute these fixed points explicitly, we have the formula

$$
\left(Q\left(N_{i}\right)(i)\right)^{\Delta^{\prime}}=\otimes_{j=i \bmod d} Q\left(N_{i}\right)[-j]
$$

where $0 \leq j \leq \ell-2$.

Example 1.3 Let $\Delta^{\prime} \subset \Delta$ be the unique subgroup of order 2, and let $N=Z_{\ell}$ with the trivial $\Delta^{\prime}$-action. Then $P(N)$ is just $S$ with the $\Delta^{\prime}$-action it inherits from $\Delta$. Hence

$$
P\left(\Delta^{\prime}, N\right)=\otimes_{i \text { even }} S[i]=H_{*} B O
$$

On the other hand if $N$ is $Z_{\ell}$ with the non-trivial $\Delta^{\prime}$-action, $P(N)=S(1)$. That is, $\Delta^{\prime}$ is now acting trivially on the factors $S[i]$ with $i$ odd, and acting as the canonical anti-automorphism on $S[i]$ for $i$ even. So in this case,

$$
P\left(\Delta^{\prime}, N\right)=\otimes_{i o d d} S[i]=H_{*} \Omega_{0}^{2} B O
$$

We now proceed to the definition of the map

$$
\phi: P\left(\Delta_{F}, \hat{K}_{0} R_{0}\right) \longrightarrow H_{*} B G L R^{+}
$$

The definition will be facilitated by some general remarks. We begin by recalling that, for any commutative ring, $B G L R^{+}$is a $K_{0} R$-module in the homotopy category of spaces. Here we say that an object $\mathrm{X}$ of a category $\mathcal{C}$ is a module over a ring $\Phi$ if $H_{0} m_{\mathcal{C}}(-, X)$ takes values in $\Phi$-modules. To define such a module structure, we need only exhibit a homomorphism $K_{0} R \rightarrow\left[B G L R^{+}, B G L R^{+}\right]$. So suppose given a finitely generated projective module $\mathrm{P}$. Choose a projective $\mathrm{Q}$ and an isomorphism $P \oplus Q \cong R^{m}$. Choose also a bijection $\mathbb{N} \times\{1, \ldots, m\} \cong \mathbb{N}$ and use it to define an isomorphism $R^{\infty} \otimes R^{m} \cong R^{\infty}$. Then let $\rho(P): B G L R^{+} \rightarrow B G L R^{+}$denote the map induced by the group homomorphism 


$$
G L R \longrightarrow A u t\left(R^{\infty} \otimes P\right) \subset A u t\left(R^{\infty} \otimes(P \oplus Q)\right) \cong G L R
$$

where the first map is tensor product with the identity of P. Note that this homomorphism is a union of homomorphisms of the form $G L_{n} R \rightarrow G L_{m n} R$.

It is easy to see that, up to weak homotopy, $\rho(P)$ is independent of the choices made. Using the techniques of [15] and [8], it is not hard to show that $\rho(P)$ is even well-defined up to actual homotopy. However we omit the proof in view of the more sophisticated approach embodied in Remark 1.8 below. Similar remarks apply to the next proposition.

Proposition 1.4 (a) $\rho(P \oplus Q) \cong \rho(P)+\rho(Q)$; (b) $\rho(P \otimes Q) \cong \rho(P) \circ \rho(Q)$ (where o denotes composition); (c) $\rho(P)$ is an H-map.

It follows that $\rho$ extends to a ring homomorphism $K_{0} R \rightarrow\left[B G L R^{+}, B G L R^{+}\right]$, also denoted $\rho$, with the property that $\rho(\alpha)$ is an H-map for each $\alpha \in K_{0} R$. Thus for any space $\mathrm{X},\left[\mathrm{X}, B G L R^{+}\right]$is a $K_{0} R$-module. We let $\alpha \star f$ denote the product of an element $\alpha$ of $K_{0} R$ with a map $f: X \rightarrow B G L R^{+}$.

Since each $\rho(\alpha)$ is an H-map, by applying homology we obtain a $K_{0} R$ module structure on $H_{*} B G L R^{+}$in the category $\mathcal{H}$. Thus we have a ring homomorphism

$$
\bar{\rho}: K_{0} R \longrightarrow \operatorname{End}_{\mathcal{H}} H_{*} B G L R^{+}
$$

In particular $\operatorname{Hom}_{\mathcal{H} \mathcal{P}}\left(S(B), H_{*} B G L R_{0}^{+}\right)$is a module over $K_{0} R_{0}$. We again write $\alpha \star f$ for the product of $\alpha \in K_{0} R_{0}$ and $f \in H_{o m} \mathcal{H} \mathcal{P}\left(S(B), H_{*} B G L R_{0}^{+}\right)$. Note that there is a canonical element $\bar{j} \in \operatorname{Hom}_{\mathcal{H} \mathcal{P}}\left(S(B), H_{*} B G L R_{0}^{+}\right)$, induced by the canonical map $B \mu \stackrel{j}{\rightarrow} B G L R_{0}^{+}$. The next proposition is immediate from the definitions.

Proposition 1.5 (a) The map $\bar{\rho}$ factors through $\hat{K}_{0} R_{0}$. In particular, if $\alpha$ has order prime to $\ell$ then $\alpha \star f$ is the zero element $($ in $\mathcal{H})$ of

$$
\operatorname{Hom}_{\mathcal{H} \mathcal{P}}\left(S(B), H_{*} B G L R_{0}^{+}\right)
$$

(b) If $\ell^{a} \alpha=0$, then $\alpha \star \bar{j}$ factors through $P_{a}$.

(c) If $\sigma \in \Delta_{F}, \sigma \circ(\alpha \star \bar{j})=(\sigma \alpha) \star(\sigma \circ \bar{j})$.

(d) If $\alpha$ lies in the reduced K-theory $\tilde{K}_{0} R_{0}$, and $\pi: B G L R_{0}^{+} \rightarrow B G L \mathbb{F}_{0}^{+}$is the reduction map, then $\pi_{*}(\alpha \star \bar{j})=0$. 
Now fix a minimal generating set $\alpha_{0}, \ldots, \alpha_{m}$ for the $Z_{\ell^{-}}$module $\hat{K}_{0} R_{0}$. We can assume that $\alpha_{0}=1$ and that for $i>0 \alpha_{i}$ has finite order $\ell^{a_{i}}$. We also can and do assume that each $\alpha_{i}$ is an eigenvector for the $\Delta_{F}$-action - say $\sigma \alpha_{i}=\omega^{k_{i}}(\sigma) \alpha_{i}$ for $\sigma \in \Delta_{F}$. Then the maps $\alpha_{i} \star \bar{j}$ combine to induce a map in $\mathcal{H P} \Delta_{F}$

$$
\phi_{0}: P\left(\hat{K}_{0} R_{0}\right)=S(B) \otimes P_{a_{1}}\left(k_{1}\right) \otimes \ldots \otimes P_{a_{m}}\left(k_{m}\right) \longrightarrow H_{*} B G L R_{0}^{+}
$$

where we have made use of Proposition $1.5 \mathrm{a}$, b, and the $\Delta_{F}$-equivariance of $\phi_{0}$ follows from Proposition 1.5 c. Taking fixed points in $\mathcal{H}$ then yields

$$
\phi: P\left(\Delta_{F}, \hat{K}_{0} R_{0}\right) \longrightarrow H_{*} B G L R^{+}
$$

The map $\phi$ depends on the choice of generators $\alpha_{i}$, but not in any significant way. In fact it is clear that if $\phi^{\prime}$ corresponds to some other choice, $\phi$ and $\phi^{\prime}$ differ by an automorphism of $P\left(\Delta_{F}, \hat{K}_{0} R_{0}\right)$ (compare the proof of Lemma 2.3 below). In particular, $\phi$ and $\phi^{\prime}$ have the same image.

Here is the main theorem:

Theorem $1.6 \phi: P\left(\Delta_{F}, \hat{K}_{0} R_{0}\right) \longrightarrow H_{*} B G L R^{+}$is a split monomorphism of $\mathcal{P}$-Hopf algebras.

Example 1.7 Take $F=\mathbb{Q}$ and $\ell=691$. The choice of $\ell$ here is deliberate; 691 is the first non-trivial numerator in the sequence $B_{n} / n$, where $B_{n}$ is the $n$-th Bernouilli number ([16], p.347). It is known that

$$
\hat{P} i c R_{0} \cong \mathbb{Z} / 691 \oplus \mathbb{Z} / 691
$$

with $\Delta_{F}=\Delta$ acting by $\omega^{-11}$ on the first summand and as $\omega^{-199}$ on the second. ( The numbers -11, -199 are derived from the table on p.350 of [16]; the rest of our assertion can be deduced from various standard results in [16].) Hence $P^{*}\left(\hat{P} i c R_{0}\right) \cong P_{1}^{*} \otimes P_{1}^{*}$ and $P^{*}\left(\Delta_{F}, \hat{P} i c R_{0}\right) \cong P_{1}^{*}[11] \otimes P_{1}^{*}[199]$. Explicitly, $P^{*}\left(\Delta_{F}, \hat{P} i c R_{0}\right)$ is a polynomial algebra on generators $x_{m}, y_{n}$ with $\left|x_{m}\right|=$ $2 m,\left|y_{n}\right|=2 n, m=11 \bmod 690$ and $n=199 \bmod 690$. The factor $\left(S^{*}\right)^{\Delta_{F}}$ is just $S^{*}[0]$, a polynomial algebra on generators $z_{n}$ of dimension $2 n(\ell-1)$. Hence Theorem 1.6 asserts that there is a retraction of $\mathcal{P}$-Hopf algebras

$$
H^{*} G L \mathbb{Z}[1 / 691] \longrightarrow S^{*}[0] \otimes P_{1}^{*}[11] \otimes P_{1}^{*}[199]
$$


Remark 1.8 There is another way to describe the $K_{0} R$-module structure on $B G L R^{+}$, that will be used in Section 4. If $X$ is a ring spectrum, then $\Omega_{0}^{\infty} X$ is a ring space. The multiplication $\Omega_{0}^{\infty} X \wedge \Omega_{0}^{\infty} X \rightarrow \Omega_{0}^{\infty} X$ is simply the adjoint of

$$
\Sigma^{\infty} \Omega_{0}^{\infty} X \wedge \Sigma^{\infty} \Omega_{0}^{\infty} X \longrightarrow X \wedge X \longrightarrow X .
$$

In particular $\Omega_{0}^{\infty} X$ is a $\pi_{0} X$-module. According to [9], p.302, in the case $X=K R$ this ring space structure agrees with the one defined in [8]. In particular the $K_{0} R$-module structures agree.

We will not consider exterior algebras in this paper; in effect we work entirely in the category $\mathcal{H}^{+}$. However for the reader's convenience we will define the exterior algebras $E_{a}$ occuring in the conjectures of the introduction. As an object of $\mathcal{H} \mathcal{P}^{*}, E_{a}$ is the exterior algebra generated by primitives $\sigma_{n, k}$ of degree $2 n \ell^{k}+1$, where $(n, \ell)=1$ and $0 \leq k<a$. The action of $\mathcal{P}$ is obtained by identifying $E_{a}$ with a sub-Hopf algebra of $H_{*} U$. Note that any object $\mathrm{E}$ of $\mathcal{H}^{-} \mathcal{P}^{*}$ admits a unique splitting as in Remark 1.2, again denoted by $E=\otimes_{i=0}^{\ell-2} E[i]$. Here $\mathrm{E}[\mathrm{i}]$ has primitives concentrated in degrees $2 \mathrm{n}-1$ with $\mathrm{n}=\mathrm{i} \bmod \ell-1$. Now suppose given a finitely-generated $Z_{\ell}$-module $\mathrm{N}$ as before, with rank s and exponent $\ell^{a_{1}}, \ldots, \ell^{a_{m}}$. Then

$$
E(N)=\left(H_{*} U\right)^{s} \otimes E_{a_{1}} \otimes \ldots \otimes E_{a_{m}}
$$

If $\mathrm{N}$ is a $\Delta^{\prime}$-module, with eigenspace decomposition $N=\oplus N_{i}$, then

$$
E\left(\Delta^{\prime}, N\right)=\otimes_{j=i \bmod d} E\left(N_{i}\right)[-j]
$$

where $0 \leq j \leq \ell-2$. For example if $\Delta^{\prime}$ has order 2 and $\mathrm{N}$ is $Z_{\ell}$ with the trivial $\Delta^{\prime}$-action,

$$
E\left(\Delta^{\prime}, N\right)=\otimes_{i \text { even }} H_{*} U[i]=H_{*} O
$$

whereas if $\mathrm{N}$ has the non-trivial action

$$
E\left(\Delta^{\prime}, N\right)=\otimes_{i o d d} H_{*} U[i]=H_{*} U / O
$$

Finally $J_{a}=P_{a} \otimes E_{a}$ and $J\left(\Delta_{F}, N\right)=P\left(\Delta_{F}, N\right) \otimes E\left(\Delta_{F}, N\right)$. 


\section{The cohomology of GLR modulo nil ele- ments}

An element $\alpha \in H^{*} G L R$ is nil if the restriction of $\alpha$ to $H^{*} G L_{n} R$ is nilpotent for all finite $\mathrm{n}$. The nil elements form an ideal $\mathcal{N}$. It will follow from the proof below that $\mathcal{N}$ is in fact a Hopf ideal.

Theorem 2.1 The map $\phi^{*}$ of Theorem 1.6 induces an isomorphism of $\mathcal{P}$ Hopf algebras

$$
H^{*} G L R / \mathcal{N} \cong P^{*}\left(\Delta_{F}, \hat{K}_{0} R_{0}\right)
$$

Proof: We may assume that $\mu_{\ell} \subset R$, since the general case follows by taking $\Delta_{F^{-}}$fixed points in $\mathcal{H}$.

Call an n-tuple $\left(P_{1}, \ldots, P_{n}\right)$ of rank one projective submodules of $R^{n}$ a complete flag if $\oplus P_{i}=R^{n}$. Two such flags $S, S^{\prime}$ are equivalent if for some (possibly different) ordering $\left(P_{i_{1}}, \ldots, P_{i_{n}}\right)$ of $\mathrm{S}$ and $\left(P_{i_{1}}^{\prime}, \ldots, P_{i_{n}}^{\prime}\right)$ of $S^{\prime}$, we have $P_{i_{j}} \cong P_{i_{j}}^{\prime}$ as R-modules for all $\mathrm{j}$. Let $G_{S}$ denote the isotropy group of $\mathrm{S}$ in $G L_{n} R$. Then $G_{S} \cong\left(R^{\times}\right)^{n}$, so $G_{S}$ contains a unique $\ell$-torus $T_{S}$ of rank n. The next lemma is essentially proved in [12], Section 14.

Lemma 2.2 The assignment $S \mapsto T_{S}$ defines a bijection

(flags in $R^{n}$, up to ordering) $\leftrightarrow\left(\right.$ maximal $\ell$-tori in $G L_{n} R$ )

and induces a bijection

(equivalence classes of flags) $\leftrightarrow$ (conjugacy classes of maximal $\ell$-tori)

A torus $\mathrm{T}$ of rank $\mathrm{k}$ in in $G L_{n} R$ determines a map $\otimes^{k} \subset H_{*} T \rightarrow H_{*} G L R$. Let $W_{T}$ denote the image of this map. Let $\mathrm{W}$ denote the linear sum of all the vector spaces $W_{T}$.

Lemma 2.3 $\operatorname{Im} \phi=W$.

Proof: For each $\alpha \in K_{0} R$, let $V_{\alpha}$ denote the image of $\alpha \star \bar{j}: S(B) \rightarrow H_{*} B G L R^{+}$. Let $V=\sum_{\mathcal{H}} V_{\alpha}$, where the sum is over all $\alpha \in K_{0} R$, and is taken in the category $\mathcal{H}$. We will show first that $V=\operatorname{Im} \phi$. 
To show that $V \subset \operatorname{Im} \phi$, it is enough to show each $V_{\alpha} \subset \operatorname{Im} \phi$. Moreover we need only check this condition on a generating set for $K_{0} R$. Hence we can assume that either $\alpha=\alpha_{i}(0 \leq i \leq m)$, in which case $V_{\alpha} \subset \operatorname{Im} \phi$ by definition, or else $\alpha$ has order prime to $\ell$, in which case $V_{\alpha}$ is zero (in $\mathcal{H}$ ) by part (a) of Proposition 1.5. Thus $V \subset \operatorname{Im} \phi$, and since the reverse inclusion is true by definition this proves $V=\operatorname{Im} \phi$.

Now we show $\mathrm{V}=\mathrm{W}$. Note that $\mathrm{W}$ is a sub-Hopf algebra of $H_{*} B G L R^{+}$that is, $\mathrm{W}$ is closed under sums, products, coproducts and a fortiori under the canonical anti-automorphism. So to show $V \subset W$ it is enough to show $\alpha \star \bar{j}(B) \subset W$ for each $\alpha=[P], P \in$ Pic R. But clearly $\alpha \star \bar{j}(B) \subset W_{T_{S}}$, where $\mathrm{S}$ is the flag $\left(P, P^{-1}\right)$ in $R^{2}$. Finally, consider a complete flag $S=$ $\left(P_{1}, \ldots, P_{n}\right)$ in $R^{n}$ with associated maximal torus $T=T_{S}$. Let $S^{\prime}$ denote the flag $\left(P_{1}, P_{1}^{-1}, \ldots, P_{n}, P_{n}^{-1}\right)$ in $G L_{2 n} R$. Then $\mathrm{T}$ is conjugate in $G L_{2 n} R$ to the subtorus of $T_{S^{\prime}}$ consisting of elements which are trivial on the factors $P_{i}^{-1}$. It follows that $W_{T} \subset \sum \mathcal{H}_{\left[P_{i}\right]}$. Hence $W \subset V$, which completes the proof.

We can now complete the proof of the theorem. If $x \in \mathcal{N}$ then $x$ annihilates $W_{T}$ (under Kronecker product) for all $\mathrm{T}$, and hence $x$ annihilates $\operatorname{Im} \phi$ by Lemma 2.3. Conversely if $x$ annihilates $\operatorname{Im} \phi$ then $x^{2}$ restricts to zero on every $\ell$-torus $\mathrm{T}$, again by Lemma 2.3. Hence for all $\mathrm{n}$, the restriction of $x$ to $G L_{n}$ is nilpotent by [12], Theorem 14.1. So $\phi^{*}$ factors through a monomorphism $H^{*} G L R / \mathcal{N} \rightarrow P^{*}\left(\hat{K}_{0} R\right)$, which by construction is a map of $\mathcal{P}$-Hopf algebras. By Theorem 1.6, this map is an isomorphism.

We conclude this section with an observation due to Quillen ([12],section 14).Call a cohomology class $y \in H^{*} G L_{n} R$ unstable if it is not in the image of the restriction map from $H^{*} G L R$.

Proposition 2.4 Suppose Pic $R$ contains nontrivial elements of order prime to $\ell$. Then for all $n \geq 2$, there exists an element $y \in H^{*} G L_{n} R$ such that $y^{k}$ is unstable for all $k$.

The proof we will give here is really no different from Quillen's original argument, but still it is enlightening to see how it fits into our context. We first need a lemma that properly belongs in the context of an AtiyahSegal-type homomorphism for representation rings, but we will take a direct approach here. Consider a complete flag $S=\left(P_{1}, \ldots, P_{n}\right)$. Each $P_{i}$ can be expressed uniquely in the form $P_{i} \cong P_{i}^{\prime} \otimes P_{i}^{\prime \prime}$, where the order of $P_{i}^{\prime}$ (resp. 
$\left.P_{i}^{\prime \prime}\right)$ is a power of $\ell$ (resp. prime to $\ell$ ). The relation $(P \otimes Q) \oplus 1 \cong P \oplus Q$ in Pic R shows that $P_{1}^{\prime} \oplus \ldots \oplus P_{n}^{\prime} \cong R^{n}$, so there is a flag $S^{\prime}=\left(P_{1}^{\prime}, \ldots, P_{n}^{\prime}\right)$.

Now observe that a complete flag $S=\left(P_{1}, \ldots, P_{n}\right)$ defines not only a maximal $\ell$-torus, but even a specific homomorphism $\theta_{S}: \mu_{\ell}^{n} \rightarrow G L_{n} R: \theta_{S}\left(\xi_{1}, \ldots, \xi_{n}\right)$ acts as $\xi_{i}$ on $P_{i}$. Let $\kappa_{S}$ denote the induced map $B \mu_{\ell}^{n} \rightarrow B G L R^{+}$.

Lemma $2.5 \kappa_{S}$ is homotopic to $\kappa_{S^{\prime}}$.

Proof: Since $\mu^{n}$ is a finite $\ell$-group, and $B G L R^{+}$has finite type, $\left[B \mu^{n}, B G L R^{+}\right]$ is an $\ell$-complete abelian group. Hence it is enough to show that for some integer $\mathrm{d}$ prime to $\ell, d \kappa_{S}$ is homotopic to $d \kappa_{S^{\prime}}$. Choose $\mathrm{d}$ prime to $\ell$ so that $\otimes^{d} P_{i}^{\prime \prime} \cong R$ for all i. Then the definition of the H-space structure on $B G L R^{+}$ as in [8] shows that $d \kappa_{S}$ factors through $B G L_{d n} R$, where it is represented by the following map f: write $R^{d n} \cong d P_{1} \oplus \ldots \oplus d P_{n}$ and classify the homomorphism $\mu^{n} \rightarrow G L_{d n} R$ defined by requiring $\left(\xi_{1}, \ldots, \xi_{n}\right)$ to act as $\xi_{i}$ on $d P_{i}$. There is a similar map $f^{\prime}$ representing $d \kappa_{S^{\prime}}$. Since $d P_{i} \cong d P_{i}^{\prime}$, there is an element $g \in G L_{n} R$ with $g\left(d P_{i}\right)=d P_{i}^{\prime}$ for all i. Hence $g \kappa_{S^{\prime}} g^{-1}=\kappa_{S}, \mathrm{f}$ is homotopic to $f^{\prime}$, and the lemma follows.

Now assume Pic $\mathrm{R}$ contains an element $[\mathrm{P}]$ of order $d>1$ with d prime to $\ell$. For $n \geq 2$, let $\mathrm{S}$ denote the flag $\left(P, P^{-1}, R, \ldots, R\right)$ in $R^{n}$. In this case $S^{\prime}$ is the "trivial" flag whose associated torus $T_{S^{\prime}}$ lies in the diagonal matrices. Then by the lemma, the restriction maps $H^{*} G L R \rightarrow H^{*} T_{S}$ and $H^{*} G L R \rightarrow H^{*} T_{S^{\prime}}$ have identical kernels. But since $T_{S}$ and $T_{S^{\prime}}$ are nonconjugate maximal $\ell$-tori in $G L_{n} R$, there is an element $y \in H^{*} G L_{n} R$ such that y restricts to zero on $T_{S^{\prime}}$ but is nonzero - even non-nilpotent - on $T_{S}$ ([12], Proposition 11.2(ii) and Remark 14.4). Evidently $y^{k}$ is unstable for all $\mathrm{k}$.

\section{Homology of the space $Y_{R}$}

The purpose of this section is to explain some of the results of [3], in particular the homology computation Theorem 3.6 below. Since these results may appear forbiddingly technical to the uninitiated, we will begin with several miscellaneous remarks that may be helpful. We suggest that the reader skim 
through these first six paragraphs at a first reading, returning to them later as necessary. An explicit numerical example is given at the end of the section.

The homotopy category of spectra. All of the results summarized below are formulated and proved in the homotopy category (i.e. the stable category) of spectra, or of spaces. For example a "commutative ring spectrum" is simply a spectrum E together with a homotopy class of maps $E \wedge E \rightarrow E$ such that the relevant unit, associativity and commutativity diagrams commute up to homotopy; similarly for module spectra. When we speak of a group $\mathrm{G}$ acting on a spectrum E, we simply mean there is given a homomorphism from $\mathrm{G}$ into the group of self-equivalences of $\mathrm{E}$. If $\mathrm{E}$ is $\ell$-complete, such a homomorphism extends to a ring homomorphism $Z_{\ell}[G] \rightarrow[E, E]$. Any decomposition of the identity of $Z_{\ell}[\mathrm{G}]$ into orthogonal idempotents yields a corresponding wedge sum decomposition of the spectrum E: if e is an idempotent, the corresponding wedge summand is simply the mapping telescope of

$$
E \stackrel{e}{\rightarrow} E \stackrel{e}{\rightarrow} E \stackrel{e}{\rightarrow} \ldots
$$

or equivalently the spectrum obtained by applying Brown representability to the functor e[-,E]. For example, the action of $\Delta$ on $\hat{\mathcal{K}}$ by $\ell$-adic Adams operations yields the Adams splitting $\hat{\mathcal{K}} \cong \bigvee_{i=0}^{\ell-2} \Sigma^{2 i} \mathcal{E}$, where $\mathcal{E}$ is a ring spectrum whose homotopy is concentrated in degrees divisible by $2(\ell-1)$.

Similar remarks apply to group actions on commutative H-spaces.

Reduction to a residue field. A prime ideal $\beta$ of $\mathrm{R}$, as well as its residue field $\mathbb{F}=R / \beta$, will be called retractible if the natural reduction map $B G L R^{+} \rightarrow B G L \mathbb{F}^{+}$is a retraction after localization at $\ell$. Such primes always exist by [5]. In fact, choose any prime $\beta$ such that $d_{\mathbb{F}}=d_{F}$ and $a_{\mathbb{F}}=a_{F}$. Then $\beta$ is retractible, and infinitely many such primes exist by the Cebotarev density theorem. Furthermore there is a canonical choice of splitting map $B G L \mathbb{F}^{+} \rightarrow B G L R^{+}[2]$; it is an H-map whose image in homology is independent of the choice of $\beta$. If $\mathbb{F}$ is retractible for $R, \mathbb{F}_{0}$ is retractible for $R_{0}$, and so on. A choice of $\mathbb{F}$ is fixed throughout.

Adams operations vs. Galois action. As noted in the introduction, there is a natural action of the group $\Gamma^{\prime}$ on $\hat{\mathcal{K}}$ via $\ell$-adic Adams operations. This extends to a canonical isomorphism $\Lambda^{\prime} \cong \hat{\mathcal{K}}^{0} \hat{\mathcal{K}}$. On the other hand we also have the cyclotomic Galois group $\Gamma_{F}^{\prime}$ associated to the number field $\mathrm{F}$, 
and a corresponding pro-group ring $\Lambda_{F}^{\prime}$. Moreover there are canonical embeddings $\Gamma_{F}^{\prime} \rightarrow \Gamma^{\prime}$ and $\Lambda_{F}^{\prime} \rightarrow \Lambda^{\prime}$, obtained from the canonical representation of $\Gamma_{F}^{\prime}$ on $\mu_{\infty}$. From this point of view, the relation between the Galois automorphisms $\Gamma_{F}^{\prime}$ and the Adams operations $\Gamma^{\prime}$ appears in a purely formal way. In fact, as we now recall, this relation arises in a deeper way from Quillen's work on the Adams Conjecture. Fix a retractible residue field $\mathbb{F}$ as above and consider the $\ell$-adic cyclotomic extension $\mathbb{F}_{\infty}$. Then $\Gamma_{F}^{\prime}$ is canonically isomorphic to $G\left(\mathbb{F}_{\infty} / \mathbb{F}\right)$. Our fixed embedding of $R_{\infty}$ in $\mathbb{C}$ defines an embedding of the $\ell$-power roots of unity of $\mathbb{F}_{\infty}$ in $\mathrm{C}$. Hence we get a "Brauer lift" map $B G L \mathbb{F}_{\infty}^{+} \rightarrow B U$ which is well-defined after $\ell$-adic completion. Furthermore, as shown by Quillen, this map is an $\ell$-adic equivalence under which the Frobenius automorphism of $B G L \mathbb{F}_{\infty}^{+}$corresponds to the Adams operation $\Psi^{q}$ on BU.

Passing to $\Delta_{F}$-fixed points. We will invariably proceed by first adjoining $\ell$-th roots of unity to $\mathrm{R}$, denoting this extension by $R_{0}$. It is then always easy to "descend" from $R_{0}$ to $\mathrm{R}$. The reason is that $\Delta_{F}$ is a finite group of order prime to $\ell$, and our spaces and spectra are always localized (and usually completed) at the prime $\ell$. For example, consider the natural action of $\Delta_{F}$ on the spectrum $K R_{0}$. Then KR is just the "homotopy fixed-point spectrum" of this action: $\mathrm{KR}=K R_{0}^{\Delta_{F}}$, where $K R_{0}^{\Delta_{F}}$ simply means the wedge summand of $K R_{0}$ associated to the idempotent of the trivial representation.

Tate twisting. Let $\mathrm{G}$ be any group equipped with a fixed homomorphism $\omega: G \rightarrow Z_{\ell}{ }^{\times}$. In our applications, $G$ will be a subgroup of $\Delta$ and $\omega$ will be the Teichmuller character. Let $X$ be an object in some category such that $\operatorname{Hom}(\mathrm{X}, \mathrm{X})$ has a natural structure of $Z_{\ell}$-module, and suppose we are also

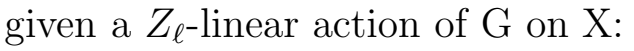

$$
\phi: Z_{\ell}[G] \longrightarrow \operatorname{Hom}(X, X)
$$

Then for any integer $\mathrm{n}$ we can define a new $Z_{\ell^{-}}$-linear action of $\mathrm{G}$ on $\mathrm{X}$, the n-th Tate twist, by

$$
\phi(n)(g)=\omega^{n}(g) \phi(g)
$$

We use the notation $\mathrm{X}(\mathrm{n})$ to denote the object $\mathrm{X}$ with this new G-action.

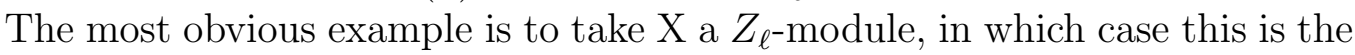
classical notion of Tate twisting; for example $Z_{\ell}(n)$ denotes the $\mathrm{n}$-th twist 
of the trivial module. However we could also take $\mathrm{X}$ to be an $\ell$-complete spectrum, an $\ell$-complete commutative $\mathrm{H}$-space, or a Hopf algebra in the category $\mathcal{H}$.

Bousfield localization. For a detailed discussion of Bousfield localization, in the context of algebraic K-theory, the reader could consult the author's expository article [11]. Here we will only make a few brief remarks. Fix a spectrum E. Then for any spectrum X, the E-localization of X consists of a spectrum $L_{E} X$ and a terminal $E_{*}$-equivalence out of $\mathrm{X}: X \rightarrow L_{E} X$. Bousfield has shown that such a functor $L_{E}(-)$ always exists; it has the effect of stripping away all information about $\mathrm{X}$ which is not visible to the homology theory E. For example if $E_{*}$ is rational homology (=rational homotopy for spectra), the homotopy groups of $L_{E} X$ are just the rationalized homotopy groups of $\mathrm{X}$. If $\mathrm{E}$ is $\bmod \ell$-homotopy, we write $\hat{X}$ for $L_{E} X$. This is (by definition) the $\ell$-adic completion of $\mathrm{X}$; $\ell$-adic completion destroys spectra with uniquely $\ell$-divisible homotopy groups. The example central to [3] is the case $E=\mathcal{K}$ or $\hat{\mathcal{K}}$; it is easy to see that the functor $L_{\hat{\mathcal{K}}}$ is just $L_{\mathcal{K}}$ followed by $\ell$-adic completion. We write $\hat{L}$ for $L_{\hat{\mathcal{K}}}$. The crucial point is that Thomason [14] has proved the Lichtenbaum-Quillen conjectures for the localized spectrum $\hat{L} K R$.

With these preliminary remarks out of the way, we turn to our summary of [3]. We begin by recalling the basic Iwasawa module $M \equiv M_{F}$, which is one of the essential ingredients of [3]. Let $E_{\infty}$ denote the maximal abelian $\ell$ extension of $F_{\infty}$ which is unramified away from $\ell$. Then $\mathrm{M}$ is the Galois group $G\left(E_{\infty} / F_{\infty}\right)$. It is an abelian pro- $\ell$-group on which $\Gamma_{F}^{\prime}$ acts (continuously) by conjugation, and hence receives a natural structure of $\Lambda_{F}^{\prime}$-module. This structure encodes a wealth of number-theoretic information ([7], [16]). We will need the following property of M:

Theorem 3.1 (Iwasawa) $M$ is a finitely-generated $\Lambda_{F}$-module, of rank $r_{2}\left(F_{0}\right)$ and of projective dimension at most one.

The next theorem is the main theorem of [3].

Theorem 3.2 There are natural isomorphisms of $\Lambda^{\prime}$-modules 


$$
\begin{aligned}
& \hat{\mathcal{K}}^{-1} K R \cong \Lambda^{\prime} \otimes_{\Lambda_{F}^{\prime}} M \\
& \hat{\mathcal{K}}^{0} K R \cong \Lambda^{\prime} \otimes_{\Lambda_{F}^{\prime}} Z_{\ell}
\end{aligned}
$$

Combining this with Iwasawa's theorem above, it follows that $\hat{\mathcal{K}}^{*} K R$ is finitely-generated as a module over $\Lambda^{\prime}$, of projective dimension at most one.

Remark 3.3 The spectrum $\hat{\mathcal{K}}$ is periodic, with period 2, so Theorem 3.2 completely describes $\hat{\mathcal{K}}^{*} K R$. Explicitly, there is an element $\beta \in \pi_{2} \hat{\mathcal{K}}$ such that multiplication by $\beta$ gives an equivalence $m_{\beta}: S^{2} \wedge \hat{\mathcal{K}} \rightarrow \hat{\mathcal{K}}$. However $m_{\beta}$ does not commute with Adams operations and indeed to a topologist this provides the quintessential example of "Tate twisting". The point is that $\pi_{2 n} \hat{\mathcal{K}} \cong Z_{\ell}(n)$ as $\Lambda^{\prime}$-module. Hence as $\Lambda^{\prime}$-modules, $m_{\beta}$ actually gives an isomorphism $\hat{\mathcal{K}}^{n+2} X(1) \cong \hat{\mathcal{K}}^{n} X$. In particular, $\hat{\mathcal{K}}^{1} K R \cong \Lambda^{\prime} \otimes_{\Lambda_{F}^{\prime}} M(-1)$, a fact that will be used below (note that the functor $\Lambda^{\prime} \otimes_{\Lambda_{F}^{\prime}}(-)$ commutes with Tate twisting, so our notation is unambiguous).

We comment briefly on the proof of the theorem. By definition, the natural map $K R \rightarrow \hat{L} K R$ induces an isomorphism on $\hat{\mathcal{K}}^{*}$. On the other hand Thomason [14] computed the homotopy groups of $\hat{L} K R$ :

$$
\begin{gathered}
\pi_{2 n} \hat{L} K R=H_{\grave{e} t}^{2}\left(R, Z_{\ell}(n+1)\right) \oplus H_{\grave{e}}^{0}\left(R, Z_{\ell}(n)\right) \\
\pi_{2 n+1} \hat{L} K R=H_{\grave{e} t}^{1}\left(R, Z_{\ell}(n+1)\right)
\end{gathered}
$$

where the $H^{0}$ term is $Z_{\ell}$ for $\mathrm{n}=0$ and is zero otherwise.

The strategy of [3] is to work backwards from the homotopy to the Ktheory. One of the crucial ingredients of this procedure is the fact that the natural map $\hat{L} K R \rightarrow \hat{L} K \mathbb{F}$ is a retraction whose right inverse is a canonical map of ring spectra. In particular $\hat{L} K R$ has a canonical structure of $\hat{L} K \mathbb{F}$ module spectrum.

Remark 3.4 Although we cannot give any details of the proof here, considerable insight can be gained by pondering why Theorem 3.2 is at least consistent with Thomason's theorem. For simplicity we assume that $R$ contains the $\ell$-th 
roots of unity. We first give an analogy. Suppose $X_{\infty} \rightarrow X$ is an infinite cyclic covering map, with group $G$. Then for any $\mathbb{Z}[G]$-module $N$ there is a natural universal coefficient spectral sequence

$$
\operatorname{Ext}_{\mathbb{Z} G}^{p}\left(H_{q}\left(X_{\infty} ; \mathbb{Z}\right) ; N\right) \Rightarrow H^{p+q}(X ; \tilde{N})
$$

where $\tilde{N}$ is the associated local coefficient system on $X$. Since $\mathbb{Z} G$ has global dimension 2, this spectral sequence collapses at $E_{3}$. Now consider the map of schemes $\operatorname{Spec}\left(R_{\infty}\right) \rightarrow \operatorname{Spec}(R)$. This can be viewed as a Galois covering whose group of deck transformations - namely $\Gamma_{F}$ - is at least topologically cyclic. Moreover one can show that there is an analogous spectral sequence in étale cohomology, which in the case of $\mathbb{Z}_{\ell}(n)$-coefficients takes the form

$$
\operatorname{Ext}_{\Lambda_{F}^{p}}\left(H_{q}^{e ́ t}\left(R_{\infty} ; \mathbb{Z}_{\ell}\right) ; \mathbb{Z}_{\ell}(n)\right) \Rightarrow H_{e ́ t}^{p+q}\left(R ; \mathbb{Z}_{\ell}(n)\right)
$$

Here $H_{q}^{e ́ t}\left(R_{\infty} ; \mathbb{Z}_{\ell}\right)$ denotes étale homology, which we will not define here. Again the spectral sequence collapses at $E_{3}$, for the same reason. However in this case the groups $H_{q}^{\text {ét }}\left(R_{\infty} ; \mathbb{Z}_{\ell}\right)$ are zero for $q>1$. Moreover $H_{0}^{e ́ t}\left(R_{\infty} ; \mathbb{Z}_{\ell}\right)=\mathbb{Z}_{\ell}$ and $H_{1}^{e ́ t}\left(R_{\infty} ; \mathbb{Z}_{\ell}\right)=M$. So the spectral sequence collapses at $E_{2}$ and we conclude that there are isomorphisms

$$
H_{e ́ t}^{2}\left(R ; \mathbb{Z}_{\ell}(n)\right) \cong \operatorname{Ext}_{\Lambda_{F}}^{1}\left(M ; \mathbb{Z}_{\ell}(n)\right)
$$

as well as (split) short exact sequences

$$
0 \rightarrow \operatorname{Ext}_{\Lambda_{F}}^{1}\left(\mathbb{Z}_{\ell}, \mathbb{Z}_{\ell}(n)\right) \longrightarrow H_{e ́ t}^{1}\left(R ; \mathbb{Z}_{\ell}(n)\right) \longrightarrow \operatorname{Hom}_{\Lambda_{F}}\left(M, \mathbb{Z}_{\ell}(n)\right) \rightarrow 0
$$

Now suppose given Theorem 3.2. We could then compute the homotopy groups of $\hat{L} K R$ using the $\hat{\mathcal{K}}$-based Adams-Novikov spectral sequence, which in this case can be written in the form

$$
E_{2}^{s, t}=\operatorname{Ext}_{\Lambda^{\prime}}^{s}\left(\hat{\mathcal{K}}^{\epsilon} K R, Z_{\ell}(j)\right) \Rightarrow \pi_{2 j+\epsilon} \hat{L} K R
$$

where $t=2 j+\epsilon$ and $\epsilon=0$ or $\epsilon=-1$. So, after an application of Shapiro's lemma, this spectral sequence leads to the results (1),(2). Hence Thomason's theorem and Theorem 3.2 are at least consistent. (Readers not familiar with the Adams spectral sequence can avoid it by using the explicit resolutions described below.) 
The retraction $\hat{L} K R \rightarrow \hat{L} K \mathbb{F}$ induces an isomorphism on $\hat{\mathcal{K}}^{0}$. So if $\hat{L} K^{\text {red }} R$ is the fibre of this retraction, $\hat{\mathcal{K}}^{0} \hat{L} K^{\text {red }} R=0$ and

$$
\hat{\mathcal{K}}^{-1} \hat{L} K^{r e d} R=\Lambda^{\prime} \otimes_{\Lambda_{F}^{\prime}} M
$$

The low projective dimension of $\mathrm{M}$ then leads immediately to an explicit description of $\hat{L} K^{\text {red }} R$. Roughly, one simply obtains a cofibre sequence

$$
\stackrel{a}{\bigvee} \hat{\mathcal{K}} \longrightarrow \bigvee^{b} \hat{\mathcal{K}} \longrightarrow \hat{L} K^{r e d} R
$$

for suitable $a, b$, in which the first map is a matrix of K-theory operations arising from a choice of length one resolution of M. However we wish to choose this resolution in a particular way.

In order to emphasize the simple, formal nature of the construction, let us extract the essential data. It is convenient to put the emphasis on $K R_{0}$, waiting until the last minute to descend to KR. Note that $\Lambda_{F}=\Lambda_{F_{0}}=\Lambda_{F_{0}}^{\prime}$. We are given

(i) a finitely-generated $\Lambda_{F}^{\prime}$-module $\mathrm{N}$ (namely, $\mathrm{M}$ ) of projective dimension at most one

(ii) a $\hat{\mathcal{K}}$-local spectrum $X_{0}$ with $\Delta_{F}$-action (namely, $\hat{L} K^{\text {red }} R_{0}$ ) with $\hat{\mathcal{K}}^{0} X_{0}=0$

(iii) an isomorphism $\hat{\mathcal{K}}^{-1} X_{0} \cong \Lambda^{\prime} \otimes_{\Lambda_{F}^{\prime}} N$ of $\Lambda^{\prime}$-modules, compatible with the $\Delta_{F}$-action on $\mathrm{X}$ and $\mathrm{N}$.

Here the fact that $\mathrm{F}$ is a number field is irrelevant; the subscript $\mathrm{F}$ only serves to specify particular subgroups $\Delta_{F}$ and $\Gamma_{F}$ of $\Gamma$, etc. We set $X=$ $X_{0}^{\Delta_{F}}$. Now choose a resolution

$$
0 \rightarrow P_{1} \rightarrow P_{0} \rightarrow N \rightarrow 0
$$

by finitely-generated projective $\Lambda_{F}^{\prime}$-modules. We will regard this as a resolution by free $\Lambda_{F}$-modules which is compatible with the $\Delta_{F}$-eigenspace decomposition of $\mathrm{N}$. Thus the exact sequence above has the form

$$
0 \longrightarrow \Lambda_{F}{ }^{b} \stackrel{\bar{C}}{\longrightarrow} \Lambda_{F}{ }^{a} \longrightarrow N \longrightarrow 0
$$


where $\bar{C}$ is a matrix of power series in $\Lambda_{F}$ and the eigenspace decomposition is understood. We will assume further that the resolution is a minimal resolution as $\Lambda_{F}$-modules, and that $\bar{C}(0)$ is a quasi-diagonal matrix - i.e., $\bar{C}_{i, j}(0)=0$ for $i \neq j$, and each $\bar{C}_{i, i}(0)$ is either zero or of the form $\ell^{d}$. Note that $\bar{C}(0)$ is a matrix over $Z_{\ell}$, and that the quasi-diagonal condition is easily arranged by multiplying $\bar{C}$ by suitable constant matrices.

Now tensor up over $\Lambda_{F}\left(\right.$ not $\Lambda_{F}^{\prime}$ !) to get a free resolution over $\Lambda^{\prime}$

$$
0 \longrightarrow\left(\Lambda^{\prime}\right)^{b} \stackrel{C}{\longrightarrow}\left(\Lambda^{\prime}\right)^{a} \longrightarrow \Lambda^{\prime} \otimes_{\Lambda_{F}} N \longrightarrow 0
$$

This is to be regarded as an exact sequence of $\Lambda^{\prime}\left[\Delta_{F}\right]$-modules. The matrix $\mathrm{C}$ is now a matrix with coefficients in $\Lambda$, and $\mathrm{C}(0)$ is still quasi-diagonal. We then obtain, in an obvious way, a fibre sequence of spectra with $\Delta_{F}$-action

$$
\stackrel{a}{\bigvee} \hat{\mathcal{K}} \stackrel{C^{t}}{\longrightarrow} \bigvee^{b} \hat{\mathcal{K}} \stackrel{\delta}{\longrightarrow} X_{0} \rightarrow \bigvee^{a} \Sigma \hat{\mathcal{K}} \longrightarrow \bigvee^{b} \Sigma \hat{\mathcal{K}}
$$

where $C^{t}$ is the transpose matrix. We emphasize that the compatibility of this sequence with the $\Delta_{F}$-action is an utter triviality; it amounts to nothing more than a wedge decomposition of the given fibre sequence into d summands, one for each character of $\Delta_{F}$.

Remark 3.5 Note that there is an exact sequence of $Z_{\ell} \Delta_{F}$-modules

$$
0 \longrightarrow \pi_{1} X \longrightarrow Z_{\ell}^{a} \stackrel{C^{t}(0)}{\longrightarrow} Z_{\ell}^{b} \longrightarrow \pi_{0} X \longrightarrow 0
$$

Let $\operatorname{rank} \pi_{1} X=e$ and write $\pi_{0} X \cong Z / \ell^{a_{1}} \oplus \ldots \oplus Z / \ell^{a_{m}} \oplus Z_{\ell}{ }^{t}$. Then because the original resolution was minimal, we conclude that $b=m+t$ and $a=e+m$.

Passing to zero-th spaces in (4) (and restricting to the basepoint component of the second and third terms) yields a fibre sequence of spaces

$$
(\hat{B U})^{a} \times \pi_{1} X \rightarrow(\hat{B U})^{b} \rightarrow \Omega_{0}^{\infty} X
$$

Applying homology and analyzing the Rothenberg-Steenrod spectral sequence yields an exact sequence in $\mathcal{H} \mathcal{P} \Delta_{F}$

$$
\otimes^{a} S(B) \rightarrow \otimes^{b} S(B) \rightarrow H_{*} \Omega_{0}^{\infty} X \rightarrow \otimes^{a} H_{*} U
$$


in which the first map is precisely $C^{t}(0)$, regarded as a matrix over $E n d_{\mathcal{H}} S(B)$. (See [10] for a discussion of $\operatorname{End}_{\mathcal{H}} S(B)$.) Since C(0) is quasi-diagonal, we may write this as

$$
1 \rightarrow P\left(\pi_{0} X_{0}\right) \rightarrow H_{*} \Omega_{0}^{\infty} X_{0} \rightarrow E \rightarrow 1
$$

where $E=\left(H_{*} \Omega_{0}^{\infty} X_{0}\right)^{-}$and the sequence splits uniquely in $\mathcal{H P} \Delta_{F}$.

Passing to $\Delta_{F}$-fixed points in $\mathcal{H}$ yields

$$
H_{*} \Omega_{0}^{\infty} X \cong P\left(\Delta_{F}, \pi_{0} X_{0}\right) \otimes E^{\Delta_{F}}
$$

Now return to the case $X_{0}=\hat{L} K^{\text {red }} R_{0}$. Here we start from a resolution as in (3) of M(-1). Then by Thomason's theorem we have

$$
\pi_{1} X_{0}=H_{e t}^{1}\left(R, Z_{\ell}(1) \cong\left(R_{0}^{\times}\right)^{\wedge} \cong Z_{\ell}^{r_{2,0}+s_{0}-1}\right.
$$

and a short exact sequence

$$
0 \rightarrow \hat{P} i c R_{0} \longrightarrow \pi_{0} \hat{L} K^{r e d} R_{0} \longrightarrow T B r R_{0} \rightarrow 0
$$

where $T B r R_{0}$ is the Tate module of the Brauer group as defined in the introduction. It is a free $Z_{\ell}$-module of rank $s_{0}-1$. Here $\pi_{0} \hat{L} K^{\text {red }} R_{0} \cong$ $H_{e ̂ t}^{2}\left(R_{0}, Z_{\ell}(1)\right)$. Writing

$$
\hat{P} i c R_{0} \cong \mathbb{Z} / \ell^{a_{1}} \oplus \ldots \oplus \mathbb{Z} / \ell^{a_{m}}
$$

we see that the numbers a and $\mathrm{b}$ defined above are given in this case by

$$
\begin{gathered}
a=m+r_{2,0}+s_{0}-1 \\
b=m+s_{0}-1
\end{gathered}
$$

Bringing the residue field $\mathbb{F}$ back into the picture, we get the following theorem ([3], Theorem 10.11):

Theorem 3.6 (Dwyer-Mitchell) There is an isomorphism in $\mathcal{H} \mathcal{P}$

$$
\begin{gathered}
H_{*} \Omega_{0}^{\infty} \hat{L} K R \cong H_{*} B G L \mathbb{F} \otimes E\left(\Delta_{F}, R_{0}^{\times} / \text {torsion }\right) \\
\otimes J\left(\Delta_{F}, \hat{P} i c R_{0}\right) \otimes J\left(\Delta_{F}, T B r R_{0}\right)
\end{gathered}
$$

where $J\left(\Delta_{F},-\right)=P\left(\Delta_{F},-\right) \otimes E\left(\Delta_{F},-\right)$ as in Section 1. 
For our present purposes, only the following corollary is relevant:

\section{Corollary 3.7}

$$
\left(H_{*} \Omega_{0}^{\infty} \hat{L} K R\right)^{+} \cong P\left(\Delta_{F}, \hat{K}_{0} R_{0}\right) \otimes P\left(\Delta_{F}, T B r R_{0}\right)
$$

To illustrate the preceeding constructions, we consider the example discussed in Section 1: $\ell=691$ and $R=\mathbb{Z}[1 / \ell]$. In this case the Iwasawa module $\mathrm{M}$, including the $\Delta$-eigenspace decomposition, can be described quite explicitly. Call a character of $\Delta$ even (resp. odd) if it is an even (resp. odd) power of the Teichmuller character $\omega$.

Proposition 3.8 $M(-1)_{\chi} \cong \Lambda$ if $\chi$ is even, and if $\chi$ is odd there are power series $f_{\chi} \in \Lambda$ such that $M(-1)_{\chi}=\Lambda / f_{\chi}$. Moreover for $\chi$ odd, $f_{\chi}$ is a unit unless $\chi=\omega^{-11}$ or $\chi=\omega^{-199}$, in which case $\nu_{\ell} f_{\chi}(0)=1$.

Here the power series $f_{\chi}$ are related to $\ell$-adic L-functions, and can be taken to be linear polynomials. The condition on the odd characters comes about because these are the only characters which occur in the eigenspace decomposition of $\hat{P} i c R_{0}$. The condition on the even characters can be traced, ultimately, to Dirichlet's unit theorem. It would require too lengthy a digression to prove these assertions here; the interested reader should consult [16], especially Chapter 13 and Theorem 10.16.

Let $f=f_{\omega^{-11}}, g=f_{\omega^{-199}}$. Since we are free to multiply each $f_{\chi}$ by a unit, we can assume $\mathrm{f}(0)=\ell=\mathrm{g}(0)$. We can take the matrix $\bar{C}$ to be the $\left(2+\frac{l-1}{2}\right) \times(2)$-matrix whose first two rows are

$$
\left(\begin{array}{ll}
f & 0 \\
0 & g
\end{array}\right)
$$

and whose remaining entries are zero. Since $a_{F}=1$ in this case, we have $C=\bar{C}$. The fibre sequence given by the first three terms of (4) breaks up as the wedge of $\frac{l-1}{2}$ fibre sequences

$$
\hat{\mathcal{K}}(i) \longrightarrow * \longrightarrow \Sigma \hat{\mathcal{K}}(i)
$$

with i even (see the remarks on Tate twisting above) and two fibre sequences

$$
\hat{\mathcal{K}}(-11) \stackrel{f}{\longrightarrow} \hat{\mathcal{K}}(-11) \longrightarrow Y
$$




$$
\hat{\mathcal{K}}(-199) \stackrel{f}{\longrightarrow} \hat{\mathcal{K}}(-199) \longrightarrow Z
$$

and hence

$$
\hat{L} K^{\text {red }} R_{0} \cong \bigvee_{i \text { even }} \Sigma \hat{\mathcal{K}}(i) \vee Y \vee Z
$$

Thus

$$
\Omega_{0}^{\infty} \hat{L} K^{r e d} R_{0} \cong\left(U^{\wedge}\right)^{345} \times \Omega_{0}^{\infty} Y \times \Omega_{0}^{\infty} Z
$$

and

$$
H_{*} \Omega_{0}^{\infty} \hat{L} K R_{0} \cong H_{*} B G L \mathbb{F}_{0} \otimes\left(H_{*} U^{\wedge}\right)^{345} \otimes J_{1} \otimes J_{1}
$$

where $J_{1}=P_{1} \otimes E_{1}$ as in Section 1 .

Finally, we descend to $\mathbb{Z}[1 / \ell]$ itself. Note that $(\hat{\mathcal{K}}(i))^{\Delta}$ is precisely the Adams summand $\mathcal{E}^{-i}$. Hence $\left(\bigvee_{\text {ieven }} \Sigma \hat{\mathcal{K}}(i)\right)^{\Delta}$ is precisely the real K-theory spectrum $K O^{\wedge}$, and so

$$
\hat{L} K^{r e d} R \cong \Sigma K O^{\wedge} \vee Y^{\Delta} \vee Z^{\Delta}
$$

where for example $Y^{\Delta}$ fits in a fibre sequence

$$
\Sigma^{11} \mathcal{E} \stackrel{f}{\longrightarrow} \Sigma^{11} \mathcal{E} \longrightarrow Y^{\Delta}
$$

and in particular the first nonvanishing positive-dimensional homotopy group of $Y^{\Delta}$ is $\pi_{22} Y^{\Delta}=\mathbb{Z} / \ell$. Similarly

$$
\Omega_{0}^{\infty} \hat{L} K^{r e d} R \cong(U / O)^{\wedge} \times \Omega_{0}^{\infty} Y^{\Delta} \times \Omega_{0}^{\infty} Z^{\Delta}
$$

and finally

$$
H_{*} \Omega_{0}^{\infty} \hat{L} K R \cong H_{*} B G L \mathbb{F} \otimes H_{*}(U / O) \otimes J_{1}[11] \otimes J_{1}[199]
$$

where $J_{a}[i]$ is defined in Section 1 . 


\section{Proof of the Retraction Theorem}

Consider the composite map $\theta$ in $\mathcal{H} \mathcal{P} \Delta_{F}$

$$
P\left(\Delta_{F}, \hat{K}_{0} R_{0}\right)=S \otimes P_{a_{1}} \otimes \ldots \otimes P_{a_{m}} \stackrel{\phi_{0}}{\longrightarrow} H_{*} B G L R_{0}^{+} \longrightarrow H_{*} \Omega_{0}^{\infty} \hat{L} K R_{0}
$$

Here $P_{a_{i}}$ corresonds to a fixed eigenvector $\alpha_{i}$ of the $\Delta_{F}$-action on $\hat{P} i c R_{0}$, and has the corresponding $\Delta_{F}$-action as in Section 1 .

We will show that $\theta$ is an isomorphism onto the corresponding summand $\mathrm{P}\left(\hat{K}_{0} R_{0}\right)$ of $H_{*} \Omega_{0}^{\infty} \hat{L} K R_{0}$ described in 3.7. This will prove Theorem 1.6 for $R_{0}$, and the general case then follows by passing to $\Delta_{F}$-fixed points in $\mathcal{H}$. Clearly it is enough to show that the image of $\theta$ is $\mathrm{P}\left(\hat{K}_{0} R_{0}\right)$. Moreover one easily reduces to checking the analogous assertion with $\hat{K}_{0} R_{0}$ replaced by $\hat{P} i c R_{0}$ (where we identify Pic $R_{0}$ with $\tilde{K}_{0} R_{0}$ in the usual way).

Extend the generating set $\alpha_{1}, \ldots, \alpha_{m}$ for $\hat{P} i c R_{0}$ to a generating set $\alpha_{1}, \ldots, \alpha_{m+t}$ for $\pi_{0} \hat{L} K^{\text {red }} R_{0}$ such that $t=s_{0}-1$ and $\alpha_{m+1}, \ldots, \alpha_{m+t}$ are also eigenvectors for the $\Delta_{F}$-action. Consider the map

$$
\delta: \bigvee^{m+t} \hat{\mathcal{K}} \rightarrow \hat{L} K^{r e d} R_{0}
$$

where $t=s_{0}-1$. Write $\delta_{i}$ for the individual maps $\hat{\mathcal{K}} \rightarrow \hat{L} K^{\text {red }} R_{0}$. We can assume that $\pi_{0} \delta_{i}$ maps the unit of $\hat{\mathcal{K}}$ to $\alpha_{i}, 1 \leq i \leq m+t$. Now let $g_{i}$ denote the composite

$$
\Sigma^{\infty} B \mu_{+} \stackrel{\eta-\epsilon}{\longrightarrow} K \mathbb{F}_{0} \longrightarrow \hat{L} K \mathbb{F}_{0} \longrightarrow \hat{\mathcal{K}} \stackrel{\delta_{i}}{\longrightarrow} \hat{L} K^{r e d} R_{0}
$$

where $\eta$ is the canonical map of ring spectra and $\epsilon$ is the composite

$$
\Sigma^{\infty} B \mu_{+} \rightarrow S^{0} \rightarrow K \mathbb{F}
$$

of the augmentation and the unit. Let $f_{i}=\Omega_{0}^{\infty} g_{i}$. Thus $f_{i}$ is the composite

$$
B \mu \longrightarrow B G L \mathbb{F}_{0}^{+} \longrightarrow \hat{B U} \stackrel{\Omega_{0}^{\infty} \delta_{i}}{\longrightarrow} \Omega_{0}^{\infty} \hat{L} K^{r e d} R_{0}
$$

where the unlabeled maps are the obvious ones. Each map $f_{i}$ then induces a map in $\mathcal{H} \mathcal{P} \Delta_{F}$

$$
\bar{f}_{i}: S \rightarrow H_{*} \hat{L} K^{r e d} R_{0}
$$


The proof of 3.6, as outlined above, shows that the summand $\mathrm{P}\left(\hat{P} i c R_{0}\right)$ is generated by the images of the maps $\bar{f}_{i}$.

There is another way to describe $f_{i}$. It was shown in [4] that $\delta_{i}$ is a map of $\hat{L} K \mathbb{F}_{0}$-module spectra. Hence the composite

$$
\hat{L} K \mathbb{F}_{0} \longrightarrow \hat{\mathcal{K}} \stackrel{\delta_{i}}{\longrightarrow} \hat{L} K^{r e d} R_{0}
$$

simply multiplies $\alpha_{i}$ by $\hat{L} K \mathbb{F}_{0}$; that is, it coincides with the composite

$$
\hat{L} K \mathbb{F}_{0}=\hat{L} K \mathbb{F}_{0} \wedge S^{0} \stackrel{1 \wedge \alpha_{i}}{\longrightarrow} \hat{L} K \mathbb{F}_{0} \wedge \hat{L} K^{r e d} R_{0} \longrightarrow \hat{L} K^{r e d} R_{0}
$$

Hence, after composing with the inclusion of $\hat{L} K^{r e d} R_{0}$ in $\hat{L} K R_{0}, g_{i}$ is simply the product of $(\eta-\epsilon)$ and $\alpha_{i}$ (in the ring spectrum structure on $\left.\hat{L} K R_{0}\right)$. Now suppose $i \leq m$, so $\alpha_{i} \in \hat{P} i c R_{0}$. Then $g_{i}$ lifts to a map $\tilde{g}_{i}$ into $K R_{0}$ - indeed $\tilde{g}_{i}$ is just $\alpha_{i}(\eta-\epsilon)$ in the ring spectrum structure on $K R_{0}$. Hence $f_{i}$ lifts to a map $\tilde{f}_{i}: B \mu \longrightarrow B G L R^{+}$. But $\tilde{f}_{i}$ is precisely the map $\alpha_{i} \star j$ described in Section 1 (see Remark 1.8). Hence the image of $\theta$ is precisely the Hopf summand $\mathrm{P}\left(\Delta_{F}, \hat{K}_{0} R_{0}\right)$, as desired.

\section{On the nil but non-nilpotent elements of $H^{*} G L R$}

Let $\mathcal{N}^{\prime}$ denote the ideal of nilpotent element in $H^{*} G L R$. Let $\mathcal{U}$ denote the Hopf kernel of the natural map $H^{*} G L R / \mathcal{N}^{\prime} \rightarrow H^{*} G L R / \mathcal{N}$. Suppose the Lichtenbaum-Quillen conjectures are true for $\mathrm{R}$, so that $\left(B G L R^{+}\right)^{\wedge} \cong$ $\Omega_{0}^{\infty} \hat{L} K^{\text {red }} R$. Then by Theorem 3.6, it would follow that

$$
\mathcal{U} \cong P^{*}\left(\Delta_{F}, T B r R_{0}\right) \cong\left(\otimes^{s_{0}-1} H^{*} B U\right)^{\Delta_{F}}
$$

In particular, $\mathcal{U}$ is trivial if and only if there is a unique prime dividing $\ell$ in $\mathcal{O}_{F_{0}}$. When there is more than one such prime, it would be very interesting to show directly that $P^{*}\left(\Delta_{F}, T B r R_{0}\right)$ is a retract of $H^{*} G L R$. For one thing, this would show that $\left(H_{*}\left(B G L R^{+}\right)^{\wedge}\right)^{+} \rightarrow\left(H_{*} \Omega_{0}^{\infty} \hat{L} K R\right)^{+}$is split surjective. This conjectural summand is also of interest because it is somehow related to the Brauer group of $\mathrm{R}$. These questions are closely related to the following conjecture. 
Conjecture 5.1 The natural $\hat{K}_{0} R$-module structure on $\left(B G L R^{+}\right)^{\wedge}$ extends to a $K_{0}^{\text {et }} R$-module structure, so that the map $\left(B G L R^{+}\right)^{\wedge} \rightarrow \Omega_{0}^{\infty} \hat{L} K^{\text {red }} R$ is a map of $K_{0}^{\text {ét }} R$-module spaces.

We recall here that $K_{0}^{e t} R \cong \pi_{0} \hat{L} K R$, and that there is a short exact sequence

$$
0 \longrightarrow \hat{P} i c R \longrightarrow K_{0}^{e ́ t} R \longrightarrow T B r R \longrightarrow 0
$$

It is clear that the conjecture would follow from the Lichtenbaum-Quillen conjectures. For $\Omega_{0}^{\infty} \hat{L} K R$ has such a module structure by Remark 1.8 and Thomason's theorem, which would then be inherited by $\left(B G L R^{+}\right)^{\wedge}$ if the Lichtenbaum-Quillen conjectures hold.

Suppose now that Conjecture 5.1 is valid, and denote the action of $\alpha \in$ $K_{0}^{e ́ t}$ on a map $f \in\left[X, \Omega_{0}^{\infty} \hat{L} K R\right]$ by $\alpha \star f$. The resulting $K_{0}^{e t}$-module structure on higher K-groups is not likely to be of any interest. Indeed consider any $\alpha \in \widetilde{K}_{0}^{e t} R=\pi_{0} \hat{L} K^{\text {red }} R$. Then it is easy to see that for any $x \in \pi_{n} \hat{L} K^{\text {red }} R$ $(n>0), \alpha \star x=0$. The point is that (see Remark 3.4) $\alpha$ has $\hat{\mathcal{K}}$-Adams filtration one, whereas $\pi_{n} \hat{L} K^{\text {red }} R$ is concentrated in Adams filtration zero (n odd) or one (n even). Alternatively, one could argue similarly using the descent spectral sequence of [14] or [1].

The action of $K_{0}^{e t}$ on homology, however, would definitely be non-trivial. Let $\beta_{1}=\alpha_{m+1}, \ldots, \beta_{t}=\alpha_{m+t}$, where $\mathrm{t}=\mathrm{s}-1$, and consider the maps $\beta_{i} \star j$ : $B \mu \rightarrow\left(B G L R^{+}\right)^{\wedge}$. These induce a map $\otimes^{t} H_{*} B U \rightarrow H_{*} B G L R^{+}$such that the composite

$$
\otimes{ }^{t} H_{*} B U \longrightarrow H_{*} B G L R^{+} \longrightarrow H_{*} \Omega_{0}^{\infty} \hat{L} K R
$$

is an isomorphism onto $\mathrm{P}(\mathrm{TBr} R)$. In particular this would show that all of $\left(H_{*} \Omega_{0}^{\infty} \hat{L} K R\right)^{+}$is a retract of $H_{*} B G L R^{+}$.

The conjectural maps $\beta_{i} \star j$, which should be constructed in some way from the Brauer group, would be of interest in their own right. Recall [6] that for any finite $\ell$-group $\mathrm{G}$, there is a natural ring homomorphism

$$
\theta_{G}:\left(I \mathcal{R}_{R} G\right)^{\wedge} \longrightarrow\left[B G,\left(B G L R^{+}\right)^{\wedge}\right]
$$

where $\mathcal{R}_{R} G$ is the representation ring of R-projective RG-modules and $I \mathcal{R}_{R} G$ is the kernel of the augmentation $\mathcal{R}_{R} G \rightarrow K_{0} R$. It follows from our homology calculations that the maps $\beta_{i} \star j$ are not in the image of $\theta_{\mu}$. Furthermore 
$\beta_{i} \star j$ cannot in any sense factor through $B G L_{n} R^{+}, n<\infty$, since any such factorization does come from a representation, by a theorem of Lannes. Thus the construction of such "homotopical $\ell$-tori" poses an interesting problem.

\section{References}

[1] Dwyer, W. and Friedlander, E., Algebraic and etale K-theory, Trans. Amer. Math. Soc. 292 (1985), 247-280.

[2] Dwyer,W., Friedlander,E., and Mitchell,S.A., The generalized Burnside ring and the K-theory of a ring with roots of unity, K-theory 6 (1992), 285-300.

[3] W. Dwyer and S. Mitchell, On the K-theory spectrum of a ring of algebraic integers, preprint 1993.

[4] W. Dwyer and S. Mitchell, On the K-theory spectrum of a smooth curve over a finite field, preprint 1994.

[5] Harris, B. and Segal, G., $K_{i}$ groups of rings of algebraic integers, Annals of Math. 101 (1975), 20-33.

[6] Hiller,H., $\lambda$-rings and algebraic K-theory, J. Pure Appl. Alg. 20 (1981), 241-266.

[7] Iwasawa,K., On $Z_{\ell}$ extensions of algebraic number fields, Ann. of Math. 98 (1973), 246-326.

[8] Loday,J.L., K-theorie algebrique et representation de groupes, Ann. Scient. Ecole Norm. Sup. 9 (1976), 309-378.

[9] May,J.P., Pairings of categories and spectra, J. Pure and Applied Alg. 19 (1980), 259-282.

[10] Mitchell,S.A., On p-adic topological K-theory, in Algebraic K-Theory and Algebraic Topology, P.G.Goerss and J.F. Jardine, editors, Kluwer Academic Publishers 1993, 197-204. 
[11] Mitchell, S.A., On the Lichtenbaum-Quillen conjectures from a stable homotopy-theoretic viewpoint, in Algebraic Topology and Its Applications, MSRI Publications 27, pp. 163-240, Springer-Verlag, New York, 1994.

[12] Quillen, D., The spectrum of an equivariant cohomology ring 2, Annals of Math. 94 (1971), 573-602.

[13] Schoeller,C., Etude de la categorie des algebres de Hopf commutatives connexes sur un corps, Manusc. Math. 3 (1970), 133-155.

[14] Thomason,R., Algebraic K-theory and etale cohomology, Ann. Scient. Ecole Norm. Sup. 13 (1985), 437-552.

[15] Wagoner, J.B., Delooping classifying spaces in algebraic K-theory, Topology 11 (1972), 349-370.

[16] Washington, L., Introduction to Cyclotomic Fields, Springer-Verlag, 1982. 